\title{
Malocclusions de classe II : bien choisir le moment du traitement pour optimiser l'effet orthopédique des appareils fonctionnels
}

\author{
Tiziano BACCETTI* \\ Aimablement traduit par Daniel KANTER \\ Department of Orthodontics, Università degli Studi di Firenze, Via del Ponte di Mezzo 46-48, 50127 Firenze, Italie
}

MOTS CLÉS :

Moment du traitement /

Malocclusion de classe II /

Appareils fonctionnels /

Maturation squelettique

\begin{abstract}
RÉSUMÉ - Le temps peut être considéré comme la quatrième dimension en orthopédie dento-faciale. Le moment du traitement peut en effet influencer de façon significative les résultats des thérapeutiques visant à produire un effet orthopédique sur les structures cranio-faciales. Des études céphalométriques, anciennes et récentes, toutes solides sur le plan méthodologique, indiquent clairement que le moment optimal pour le traitement des décalages squelettiques de classe II à l'aide d'un appareil fonctionnel (par exemple, le twin block) se situe pendant ou légèrement après le pic de croissance mandibulaire. Ce dernier peut être mis en évidence par un indicateur biologique de maturité squelettique individuelle fiable, tel que la méthode de la maturation vertébrale cervicale.
\end{abstract}

\begin{abstract}
Improving the effectiveness of functional jaw orthopedics in Class II malocclusion by appropriate treatment timing. Time can be considered the fourth dimension in dento-facial orthopedics. Treatment timing can play a significant role in the outcomes of treatment aimed to produce an orthopedic effect in the craniofacial structures. The results of methodologically-sound cephalometric studies of both the past and the recent history of orthodontics clearly indicate that optimal treatment timing for Class II skeletal disharmony with a functional appliance (e.g. twin block) is during or slightly after the peak in mandibular growth as revealed by a reliable biologic indicator of individual skeletal maturity such as the cervical vertebral maturation method.
\end{abstract}

KEYWORDS:

Treatment timing /

Class II malocclusion /

Functional appliances /

Skeletal maturation
Le problème du moment du traitement en orthopédie dento-faciale amène implicitement à distinguer entre traitements «précoce » et « tardif». Trop souvent, des variables floues et prêtant à confusion, telles que le stade de dentition, ont été utilisées à tort pour caractériser ces deux types de traitement. Il y a dix ans, le College of Diplomates de l'American Board of Orthodontics a publié le consensus suivant à propos de la définition d'un «traitement précoce»: «...traitement commencé en denture déciduale ou en denture mixte, qui vise à améliorer le développement squelettique et dentaire avant l'éruption des dents permanentes » [4]. Une telle assertion peut néanmoins s'avérer problématique lorsqu'elle est reliée au moment du traitement orthopédique/fonctionnel d'une 
malocclusion de classe II. En effet, le but majeur des appareils fonctionnels étant d'améliorer le « développement squelettique » mandibulaire, ce type de thérapeutiques peut sembler satisfaire la définition précédente du traitement précoce. Pourtant, le plus souvent, ces appareils fonctionnels sont mis en place trop tôt, à un moment qui n'est pas favorable d'un point de vue biologique.

Malgré les résultats de certaines études cruciales des trente dernières années $[5,6,9,10,12-15]$, les aspects biologiques relatifs à la maturation squelettique individuelle ont été négligés ou mal interprétés en ce qui concerne l'appréciation du moment optimal du traitement des insuffisances mandibulaires.

Des considérations pratiques semblent être la principale raison du recours limité aux indicateurs biologiques dans le cadre d'un exercice de routine. En effet, les courbes de croissance staturale et les examens radiographiques de la main et du poignet, qui représentent deux exemples d'utilisation d'indicateurs biologiques, requièrent un effort supplémentaire considérable de la part du clinicien. La construction d'une courbe individuelle de croissance staturale demande la répétition de plusieurs mesures (au moins tous les trois mois) pendant une longue période, tandis que l'évaluation des stades de maturation de la main et du poignet nécessite une exposition radiographique supplémentaire pour les patients.

Une approche globale du diagnostic et du traitement des malocclusions de classe II intègre aussi une évaluation des bénéfices de la thérapeutique fonctionnelle sur l'esthétique faciale. La convexité du profil observée au niveau des tissus mous étant la caractéristique principale de nombreuses malocclusions de classe II, un objectif de traitement est d'améliorer l'apparence du visage, précisément en réduisant la convexité du profil. Cette dernière considération est loin d'être secondaire puisque l'esthétique du visage a d'importantes répercussions psychologiques et sociales.

Lobjectif du présent article est de souligner l'importance d'une évaluation précise de la maturité squelettique individuelle au début du traitement orthopédique/fonctionnel. Le but est de proposer une démarche qui soit à la fois facile à mettre en ouvre et efficace pour stimuler la croissance mandibulaire, indépendamment de l'âge, du stade de dentition, et, surtout, permettant d'éviter des effets indésirables

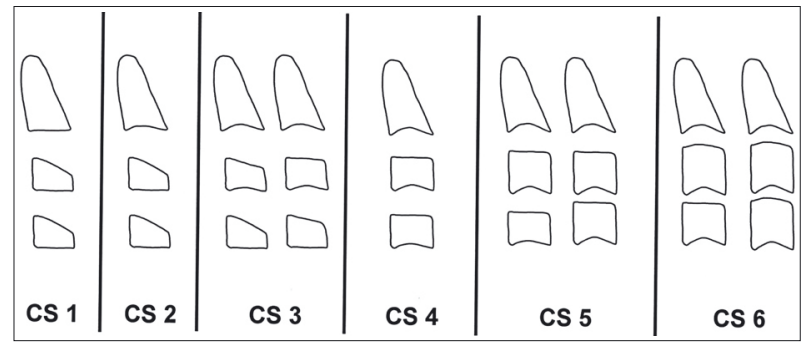

Figure 1

Stades de développement des vertèbres cervicales.

liés à la négligence des principes biologiques. La méthode de la maturation vertébrale cervicale (CVM pour Cervical Vertebral Maturation) donne une indication fiable de la maturation squelettique mandibulaire. Cette méthode est ici appliquée dans une étude qui vise à définir le moment optimal du traitement d'une malocclusion de classe II par un appareil fonctionnel, le twin block.

\section{2. Évaluation de la maturité squelettique individuelle par la méthode de la maturation vertébrale cervicale (CVM)}

Un indicateur radiographique idéal pour évaluer la maturité squelettique individuelle devrait présenter les caractéristiques suivantes: (1) La méthode doit être valide sur le plan biologique. Les informations récoltées doivent concorder avec celles obtenues par un indicateur de référence tel que la croissance staturale; (2) Il doit être efficace pour détecter le pic de croissance mandibulaire; (3) Il ne devrait pas nécessiter d'exposition radiographique en plus de la téléradiographie de profil requise pour le diagnostic et le plan de traitement orthodontiques. Les résultats d'une étude précédente de notre groupe de recherche [3] ont démontré la validité des stades de la maturation vertébrale cervicale pour l'évaluation de la maturité squelettique individuelle conformément aux précédents critères.

Six stades correspondant à six étapes de la maturation des vertèbres cervicales peuvent être mis en évidence pendant la période pubertaire [7]. Les six stades (CS pour Cervical Stages) sont caractérisés par des modifications nettes de la morphologie et de la dimension des corps vertébraux de la deuxième à la sixième vertèbre cervicale (Fig. 1). Cette méthode a montré son efficacité et sa fiabilité clinique pour 

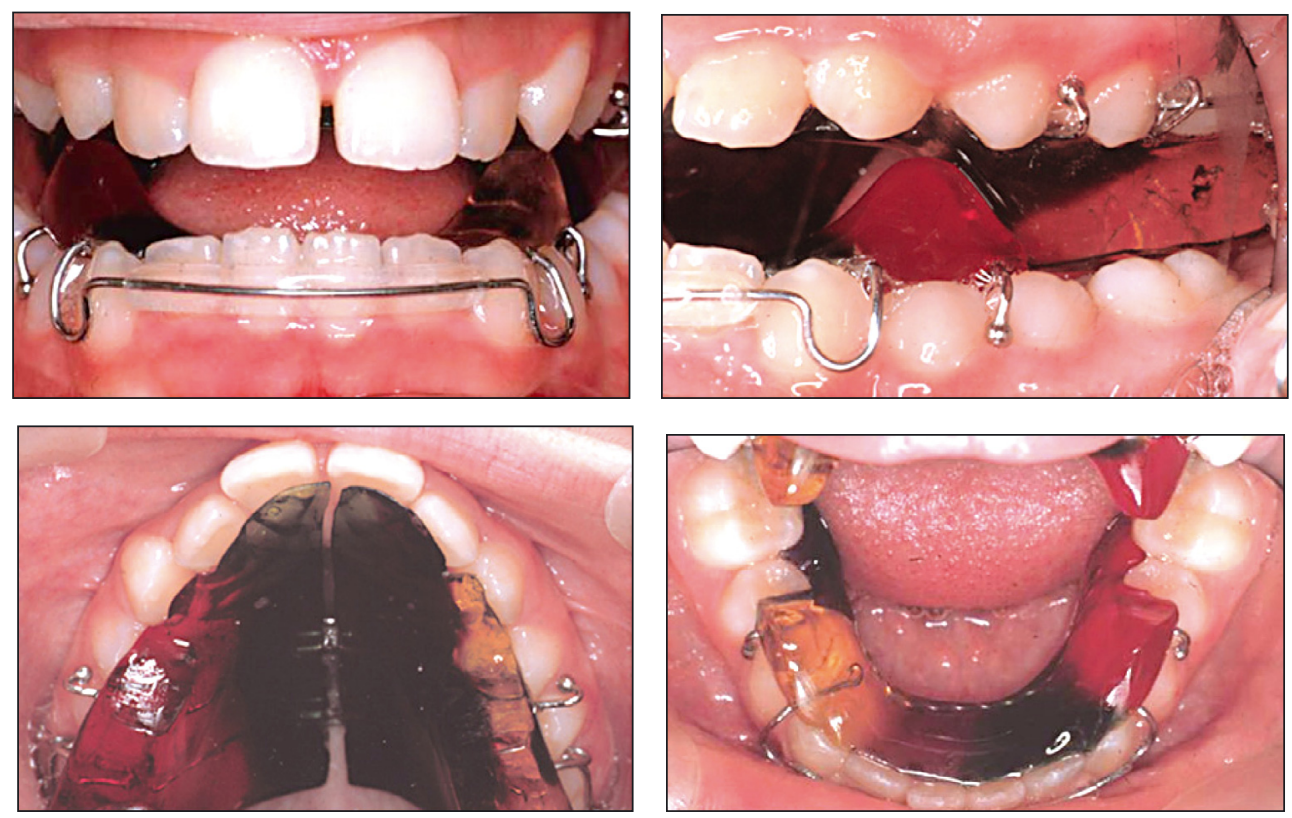

Figure 2

L'appareil twin block.

l'évaluation de la maturation squelettique mandibulaire chez des sujets en croissance. En effet, les stades de la maturation vertébrale cervicale sont corrélés aux modifications de la croissance mandibulaire qui ont lieu à la puberté $[3,11]$.

Stade 1 (CS 1): Les bords inférieurs des corps de l'ensemble des vertèbres cervicales sont plats. Les bords supérieurs sont effilés de l'arrière vers l'avant.

Stade 2 (CS 2) : Une concavité se forme au niveau du bord inférieur de la seconde vertèbre. La hauteur antérieure des corps vertébraux augmente.

Stade 3 (CS 3) : Une concavité se forme au niveau du bord inférieur de la troisième vertèbre.

Stage 4 (CS 4): Une concavité se forme au niveau du bord inférieur de la quatrième vertèbre. Des concavités commencent à se former au niveau des bords inférieurs des cinquième et sixième vertèbres. Tous les corps vertébraux cervicaux sont rectangulaires, plus larges que hauts.

Stade 5 (CS 5): Des concavités marquées apparaissent au niveau des bords inférieurs des six vertèbres cervicales. Les corps vertébraux sont presque carrés et les espaces intervertébraux sont réduits.

Stade 6 (CS 6): Toutes les concavités apparaissent plus profondes. Les corps vertébraux sont alors plus hauts que larges.

La méthode CVM présentée ici [3] est une modification et un développement du protocole original de Lamparski [7]. Plusieurs améliorations ont été apportées à la méthode originale: (1) Les stades CVM sont totalement indépendants de l'âge chronologique; (2) Les modifications de taille et de morphologie vertébrales qui caractérisent les six stades sont identiques pour les deux sexes; (3) Le pic de croissance staturale et le pic de croissance mandibulaire ont lieu tous les deux entre CS 3 et CS 4 pour $93,5 \%$ des sujets. CS 1 a lieu au moins deux ans avant le pic, CS 2 un an avant le début du pic, CS 5 un an après la fin de la portion croissante du pic, et CS 6 au moins deux ans plus tard, marquant ainsi la fin de la croissance pubertaire.

\section{Choix du moment optimal pour le traitement d'une malocclusion de classe II avec un appareil fonctionnel, le twin block}

Afin de préciser le moment favorable pour un traitement par appareils fonctionnels, cette étude s'est intéressée aux modifications squelettiques mandibulaires produites par le twin block (Fig. 2) dans deux groupes de sujets présentant un décalage de classe II et traités à différents stades de maturité squelettique mandibulaire (avant et pendant le pic pubertaire de croissance mandibulaire), cette dernière étant déterminée sur la base de la méthode CVM [2]. 


\subsection{Sujets}

Les téléradiographies de profil de 36 patients traités avec un twin block dans sept cabinets d'orthodontie privés ainsi que dans le Département d'Orthodontie de l'Université du Michigan ont été rassemblés [17]. Les praticiens devaient envoyer les radiographies avant et après traitement pour tous les patients traités avec un twin block, quels que soient les résultats thérapeutiques ou la coopération du patient. Les patients traités ont été divisés en deux groupes en fonction de leur maturité squelettique au début du traitement, grâce à la méthode de la maturation vertébrale cervicale.

Le groupe traité précocement (ETG pour earlytreated group) comptait 21 sujets (11 filles et 10 garçons). Lâge moyen de ce groupe était de 9 ans \pm 11 mois au temps $t 1$ ( $t 1$, immédiatement avant le traitement), et 10 ans, 2 mois \pm 4 mois au temps $t 2$ ( $t 2$, immédiatement après le retrait du twin block). La durée moyenne du traitement entre $t 1$ et $t 2$ était d' 1 an, 2 mois \pm 4 mois. Les stades de maturation vertébrale cervicale variaient de CS 1 à CS 2 à $t 1$, de CS 1 à CS 3 à t2. Ainsi, pour ce groupe traité précocement, le traitement a été réalisé avant le pic de croissance pubertaire.

Le groupe traité tardivement (LTG pour latetreated group) comptait 15 sujets (6 filles et 9 garçons). Lâge moyen de ce groupe était de 12 ans, 11 mois \pm 1 an, 2 mois au temps $t 1$, et 14 ans, 4 mois \pm 1 an, 3 mois au temps t2. La durée moyenne du traitement entre $t 1$ et $t 2$ était d' 1 an, 5 mois \pm 5 mois. Les stades de maturation vertébrale cervicale variaient de CS 3 à CS 5 à $t 1$, de CS 4 à CS 6 à t2. Ainsi, pour ce groupe traité tardivement, le traitement a été mis en œuvre pendant ou légèrement après le début du pic de croissance pubertaire.

Les sujets traités ont été comparés à un échantillon contrôle de 30 individus présentant des malocclusions de classe II non traitées, provenant des dossiers de l'Étude de Croissance de l'Université du Michigan (UMGS pour University of Michigan Growth Study), réalisée dans des écoles primaires et secondaires. L'échantillon contrôle a lui aussi été divisé en deux groupes en fonction du stade de maturation vertébrale cervicale.

Le groupe contrôle précoce (ECG pour early control group) comptait 16 sujets (9 filles, 7 garçons). Lâge moyen de ce groupe était de 9 ans, 1 mois \pm 10 mois à $t 1$ et 10 ans, 5 mois \pm 9 mois à $t 2$. La durée d'observation moyenne $(t 1-t 2)$ était d'l an, 4 mois \pm 7 mois. Comme pour le groupe traité précocement, les stades de maturation vertébrale cervicale variaient de CS 1 à CS 2 à $t 1$, de CS 1 à CS 3 à t2. Ainsi, le pic de croissance pubertaire n'était inclus dans la période d'observation pour aucun des sujets du groupe contrôle précoce.

Le groupe contrôle tardif (LCG pour late control group) comptait 14 sujets ( 7 filles, 7 garçons). Lâge moyen de ce groupe était de 13 ans, 7 mois \pm 1 an, 2 mois à $t 1$, et 14 ans, 10 mois \pm 1 an, 4 mois à $t 2$. La durée d'observation moyenne $(t 1-t 2)$ était d'l an, 3 mois \pm 5 mois. Comme pour le groupe traité tardivement, les stades de maturation vertébrale cervicale variaient de CS 3 à CS 5 à $t 1$, de CS 4 à CS 6 à t2. Ainsi, le pic de croissance pubertaire était inclus dans la période d'observation pour tous les sujets du groupe contrôle tardif.

\subsection{Analyse céphalométrique et analyse statistique}

Les tracés céphalométriques à $t 1$ et $t 2$ pour les deux groupes traités et les deux groupes contrôles ont été standardisés au même agrandissement et analysés par une tablette de numérisation (NumoniCS, Lansdale, PA) et un logiciel de numérisation (Viewbox, v. 3.1, dHal, Kifissia, Grèce)

Les mesures mandibulaires suivantes ont été réalisées sur tous les tracés à $t 1$ et $t 2$ : Co-Pg, Co-Go, Go-Pg, Ar-Goi-Me, CL-ML (CL étant la ligne condylaire passant par Co et le centre de la tête du condyle, ML la ligne mandibulaire passant par Go et Me).

Les variations des mesures céphalométriques entre $t 1$ et $t 2$ ont été annualisées pour les groupes traités et contrôles. Les variations annuelles du groupe traité précocement ont été comparées à celles du groupe contrôle précoce. De même, les variations annuelles du groupe traité tardivement ont été comparées à celles du groupe contrôle tardif. Lanalyse statistique a consisté en un test non-paramétrique pour des groupes indépendants (Mann-Whitney U Test, avec $p<0,05)$ grâce à un système statistique adapté (SPSS for Windows, rel. 12.0.0, SPSS, Inc.).

\subsection{Résultats}

\subsubsection{Effets du traitement dans le groupe traité précocement (Tab. 1)}

La longueur mandibulaire totale (Co-Pg) a montré une croissance significativement plus importante 
Tableau 1

Étude twin block : variations des mesures céphalométriques entre $t 1$ et $t 2$ pour les groupes précoces et tardifs. $S=c o m p a r a i s o n$ entre le groupe traité et le groupe contrôle statistiquement significative avec $p<0,05$; NS = comparaison non significative.

\begin{tabular}{|c|c|c|c|c|c|c|c|}
\hline \multirow[t]{2}{*}{ Variables } & & \multicolumn{2}{|c|}{$\begin{array}{l}\text { Groupe traité précocement } \\
\qquad(\text { ETG }) \\
(n=21)\end{array}$} & \multicolumn{2}{|c|}{$\begin{array}{c}\text { Groupe contrôle précoce } \\
\qquad(\mathrm{ECG}) \\
(n=16)\end{array}$} & \multirow[t]{2}{*}{$\begin{array}{l}\text { Mann-Whitney } \\
\text { U Test }\end{array}$} & \multirow{2}{*}{$\begin{array}{l}\text { Différence entre } \\
\text { les groupes } \\
\text { ETG/ECG } \\
\text { (« effet du traitement } \\
\text { précoce») }\end{array}$} \\
\hline & & Moyenne & $\begin{array}{l}\text { Écart } \\
\text { type }\end{array}$ & Moyenne & $\begin{array}{l}\text { Ecart } \\
\text { type }\end{array}$ & & \\
\hline Co-Pg & $\mathrm{mm}$ & $+4,95$ & 2,43 & $+3,07$ & 1,09 & $S$ & $+1,88$ \\
\hline Co-Go & $\mathrm{mm}$ & $+1,98$ & 1,50 & $+1,70$ & 1,47 & NS & $+0,28$ \\
\hline Go-Pg & $\mathrm{mm}$ & $+2,98$ & 2,26 & $+1,94$ & 1,00 & NS & $+1,04$ \\
\hline CL-ML & & $+0,67$ & 0,38 & $-0,29$ & 1,28 & NS & $+0,96$ \\
\hline Ar-Goi-Me ${ }^{\circ}$ & $+0,53$ & 2,09 & $-1,32$ & 2,08 & S & $+1,85$ & \\
\hline \multirow{2}{*}{\multicolumn{2}{|c|}{ Variables }} & \multicolumn{2}{|c|}{$\begin{array}{c}\text { Groupe traité tardivement } \\
\text { (LTG) } \\
(n=15)\end{array}$} & \multicolumn{2}{|c|}{$\begin{array}{c}\text { Groupe contrôle } \\
\text { tardif } \\
(\mathrm{LCG}) \\
(n=14)\end{array}$} & \multirow[t]{2}{*}{$\begin{array}{l}\text { Mann-Whitney } \\
\text { U Test }\end{array}$} & \multirow[t]{2}{*}{$\begin{array}{l}\text { Différence entre } \\
\text { les groupes } \\
\text { LTG/LCG } \\
\text { (« effet du traitement } \\
\text { tardif ») }\end{array}$} \\
\hline & & Moyenne & $\begin{array}{l}\text { Ecart } \\
\text { type }\end{array}$ & Moyenne & $\begin{array}{l}\text { Ecart } \\
\text { type }\end{array}$ & & \\
\hline Co-Pg & $\mathrm{mm}$ & $+7,29$ & 2,39 & $+2,54$ & 1,01 & $\mathrm{~S}$ & $+4,75$ \\
\hline Co-Go & $\mathrm{mm}$ & $+3,98$ & 2,24 & $+1,25$ & 1,45 & S & $+2,73$ \\
\hline Go-Pg & $\mathrm{mm}$ & $+3,23$ & 1,24 & $+1,57$ & 1,14 & S & $+1,66$ \\
\hline CL-ML & $\circ$ & $+1,53$ & 1,33 & $-1,27$ & 2,05 & S & $+2,80$ \\
\hline Ar-Goi-Me & $\circ$ & $+0,82$ & 1,71 & $-1,03$ & 2,02 & S & $+1,85$ \\
\hline
\end{tabular}

dans le groupe traité précocement que dans le groupe contrôle précoce, alors que la hauteur du ramus (Co-Go) et la longueur du corps mandibulaire (Go-Pg) n'ont pas présenté de différence significative. Laugmentation de l'angle goniaque (Ar-Goi-Me) dans le groupe traité précocement était significative par rapport au groupe contrôle précoce. Linclinaison de la ligne condylaire par rapport à la ligne mandibulaire (CL-ML) n'était pas significativement différente d'un groupe à l'autre

\subsubsection{Effets du traitement dans le groupe traité tardivement (Tab. 1)}

Le traitement tardif, pendant ou légèrement après le pic pubertaire, a induit une croissance significativement plus importante que dans le groupe contrôle tardif pour la longueur mandibulaire totale (Co-Pg), la hauteur du ramus (Co-Go) et la longueur du corps mandibulaire (Go-Pg). Les augmentations de l'inclinaison de la ligne condylaire par rapport à la ligne mandibulaire (CL-ML) et de l'angle goniaque (Ar-Goi-Me) étaient significativement plus importantes dans le groupe traité tardivement que dans le groupe contrôle tardif.

\subsection{Discussion des résultats du traitement}

Un traitement tardif, avec le twin block mis en place pendant ou légèrement après le début du pic de croissance mandibulaire, est plus efficace qu'un traitement réalisé avant le pic, puisqu'il induit des modifications squelettiques mandibulaires plus favorables. En effet, dans cette étude, la quantité d'élongation mandibulaire supplémentaire - induite par le traitement - dans le groupe traité tardivement $(4,75 \mathrm{~mm} / \mathrm{an})$ était plus de deux fois supérieure à celle du groupe traité précocement $(1,88 \mathrm{~mm} / \mathrm{an})$. Cette augmentation plus importante de la longueur mandibulaire totale (Co-Pg) était associée avec des augmentations supplémentaires significatives de la hauteur du ramus (Co-Go, 2,73 mm/an) et de la longueur du corps de la mandibule (Go-Pg, $1,66 \mathrm{~mm} / \mathrm{an}$ ) dans le groupe traité tardivement, comparé au groupe contrôle tardif. Pour le groupe traité précocement, les effets du traitement sur la hauteur 


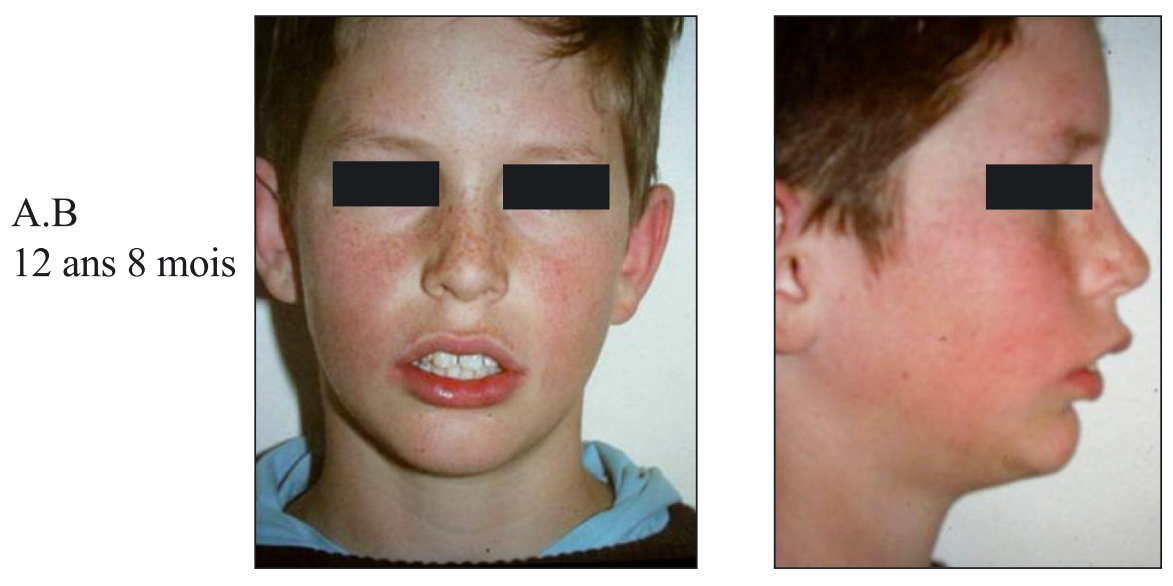

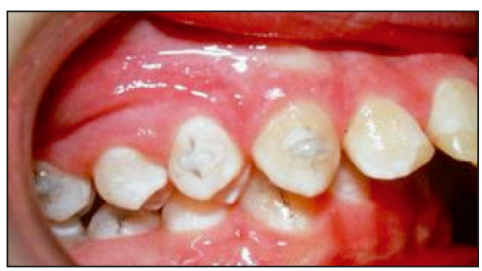

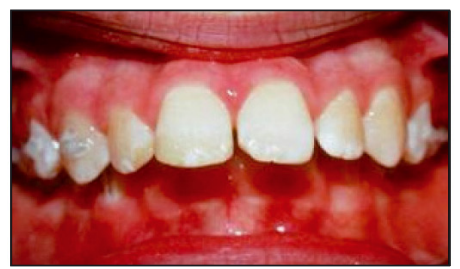

Figure 3

Photographies du visage et de l'occlusion avant le traitement avec le twin block (à 12 ans 8 mois).

$\mathrm{du}$ ramus et sur la longueur du corps de la mandibule n'étaient pas significatifs.

De plus, la croissance mandibulaire additionnelle plus importante retrouvée dans le groupe traité tardivement concordait avec des modifications significatives de la direction de croissance condylaire. Les individus traités lors du pic ont montré une direction de croissance condylaire significativement plus postérieure, illustrée par l'ouverture significative de l'angle formé par la ligne condylaire et la ligne mandibulaire (CL-ML, 2,8\%an). Cette modification de croissance a été décrite comme une « rotation mandibulaire morphogénétique postérieure » [8], un mécanisme biologique qui permet des augmentations plus importantes de la longueur mandibulaire totale, donc une amélioration efficace des rapports squelettiques sagittaux dans les malocclusions de classe II.

\section{Remarques finales}

Lune des plus grandes controverses actuelles en orthodontie porte sur le traitement précoce par appareils fonctionnels pour les patients présentant un décalage squelettique de classe II, qui figurerait l'option thérapeutique la plus favorable. Les résultats d'études céphalométriques solides sur le plan méthodologique indiquent clairement que le moment optimal du traitement d'un décalage squelettique de classe II avec un appareil fonctionnel (par exemple le twin block) se situe pendant ou légèrement après le pic de croissance mandibulaire, mis en évidence par un indicateur biologique de maturité squelettique individuelle fiable telle que la méthode de la maturation vertébrale cervicale (voir le cas clinique rapporté dans les figures 2 à 6 ).

Une phase de traitement précoce peut tout de même être mise en œuvre en début de denture mixte chez des patients présentant une malocclusion de classe II. L'objectif d'une telle intervention devrait alors se limiter à la correction de l'insuffisance transversale de l'arcade maxillaire. En effet, l'insuffisance de largeur maxillaire, à la fois au niveau occlusal et au niveau squelettique, est un aspect majeur d'une malocclusion de classe II en évolution [1] : un patient en classe II en denture mixte présente généralement un décalage transversal de 3$5 \mathrm{~mm}$ entre le maxillaire et la mandibule. Un second objectif légitime d'intervention précoce chez les sujets présentant une classe II est la correction d'une proalvéolie maxillaire avec diastème, qui peut conduire à une majoration du risque traumatique pour les incisives [18]. En revanche, le traitement destiné à induire une réponse squelettique au niveau 

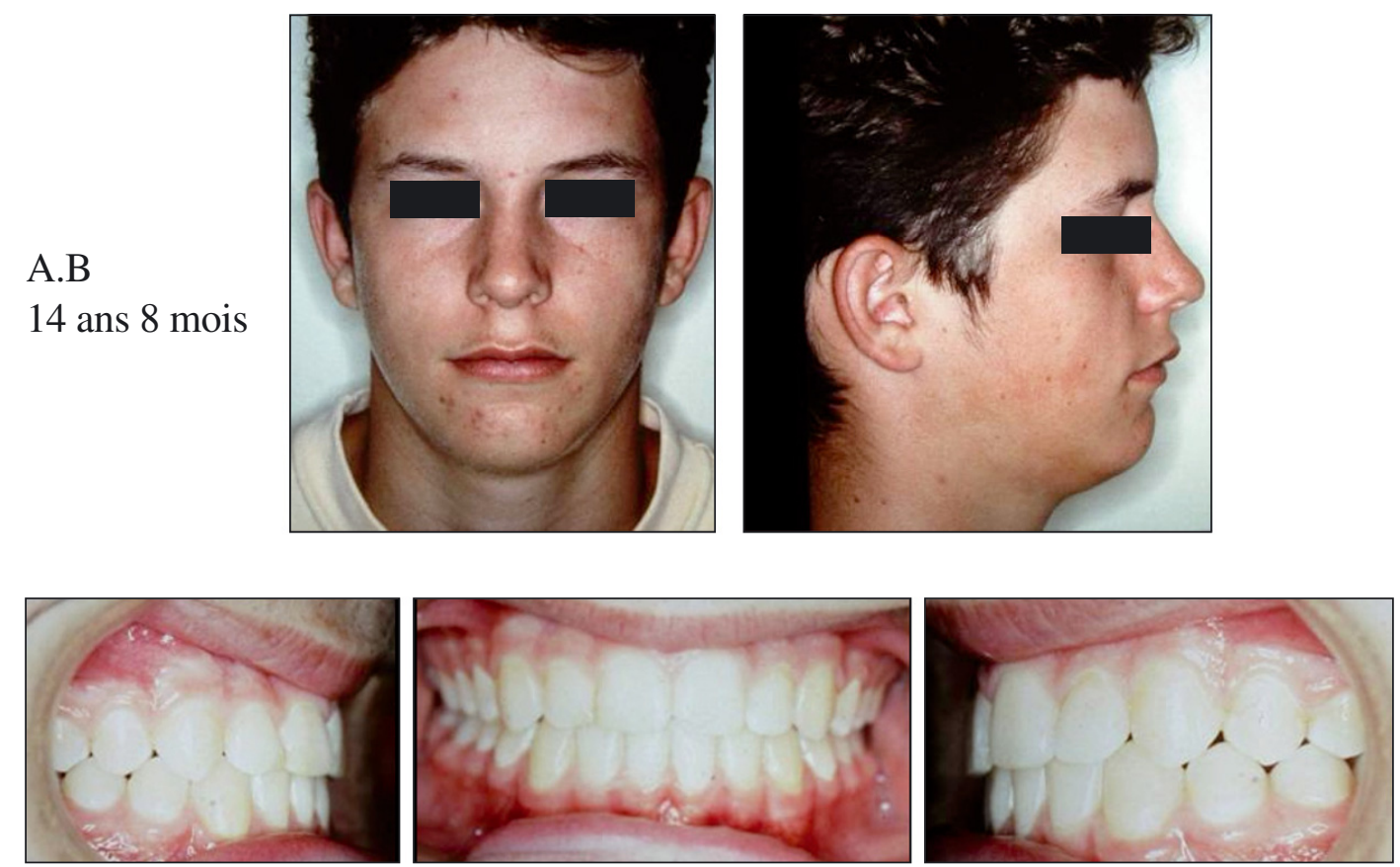

Figure 4

Photographies du visage et de l'occlusion en fin de traitement (après 12 mois de twin block et 12 mois d'appareil multi-attache, à 14 ans 8 mois).

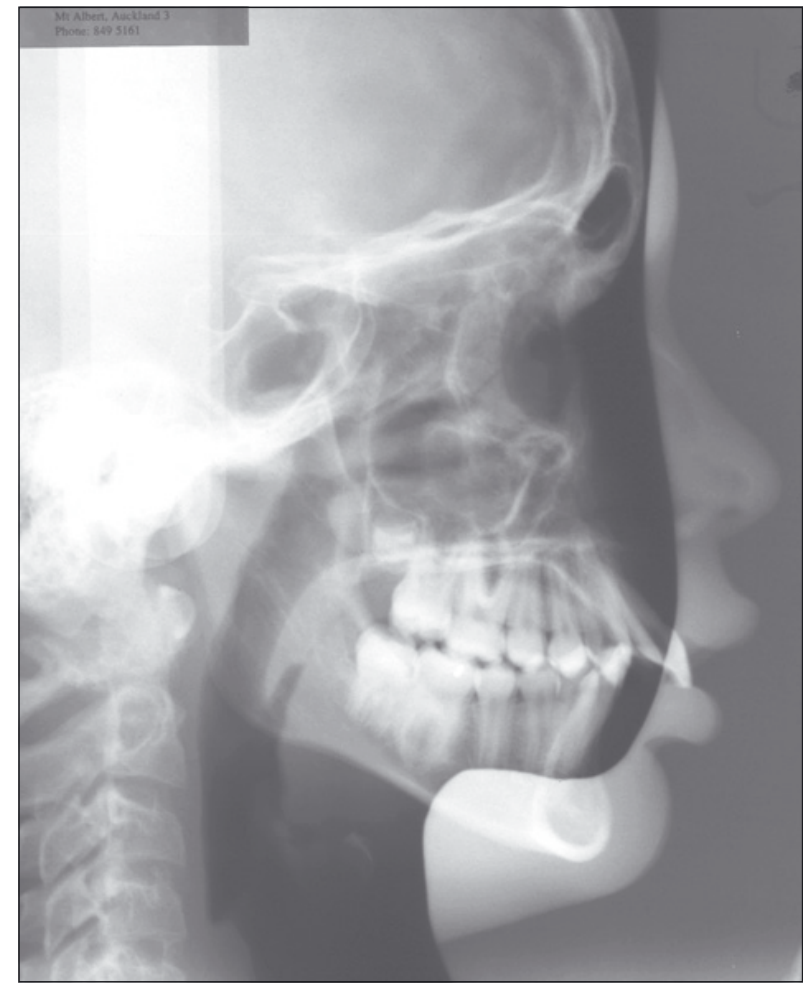

Figure 5

Téléradiographie de profil avant le traitement, montrant un stade CS 3 des vertèbres cervicales : le patient est au pic de croissance pubertaire.

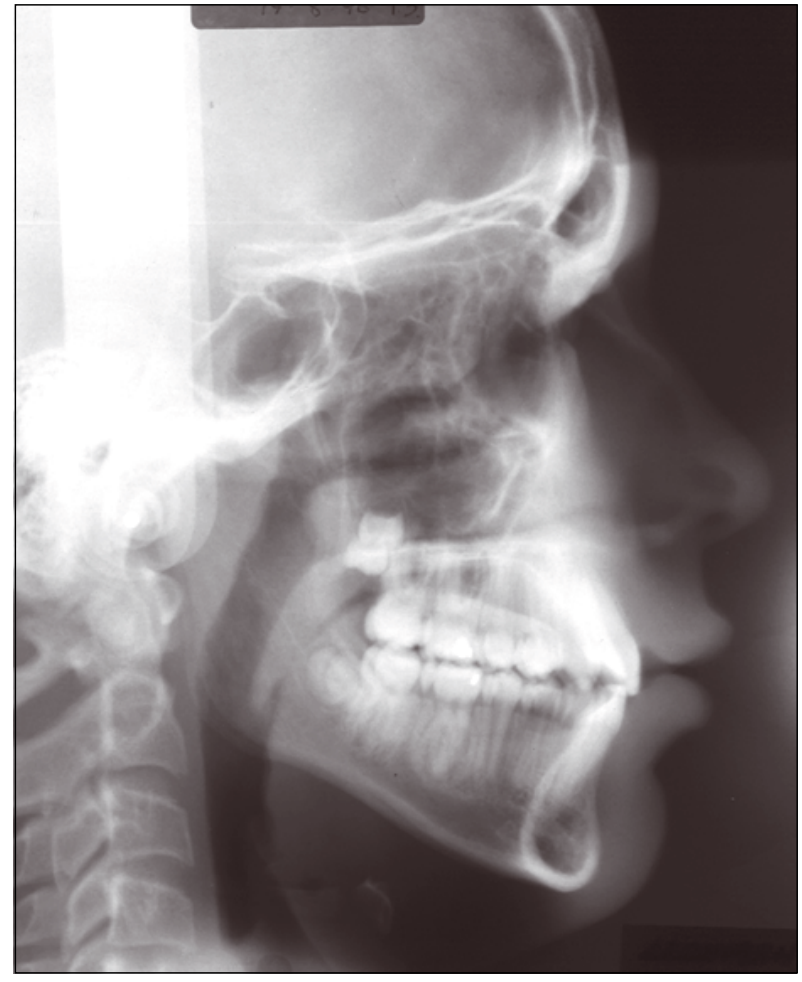

Figure 6

Téléradiographie de profil en fin de traitement (après 12 mois de twin block et 12 mois d'appareil multi-attache). 
de la mandibule, par appareil fonctionnel ou par tout autre dispositif visant un objectif thérapeutique similaire, devra, pour sa part, être reporté au début du pic de croissance pubertaire.

\section{Remerciements}

Des remerciements particuliers vont à Michael Powell pour sa révision de la version anglaise du manuscrit et au Dr Forbes Leishman qui a fourni le cas traité par twin block (Fig. 3-5). Cet article est dédié à la mémoire d'Alexander Petrovic, enseignant, mentor et ami.

\section{Bibliographie}

[1] Baccetti T, Franchi L, McNamara JA Jr, Tollaro I, Early dentofacial features of Class II malocclusion: a longitudinal study from the deciduous through the mixed dentition. Am J Orthod Dentofacial Orthop 1997;111:502-509.

[2] Baccetti T, Franchi L, Toth LR, McNamara JA Jr. Treatment timing for twin block therapy. Am J Orthod Dentofac Orthop 2000;118:159-170.

[3] Baccetti T, Franchi L, McNamara JA Jr. The cervical vertebral maturation (CVM) method. Semin Orthod 2005;11:119-129.

[4] Bishara SE, Justus R, Graber TM. Proceedings of the workshop discussions on early treatment held by the College of Diplomates of the American Board of Orthodontics in Quebec City, Canada, on July 13-17, 1997. Am J Orthod Dentofacial Orthop 1998;113:5-6.

[5] Hägg U, Pancherz H. Dentofacial orthopaedics in relation to chronological age, growth period and skeletal development. An analysis of 72 male patients with Class II, division 1 malocclusion treated with the Herbst appliance. Eur J Orthod 1988;10:169-176.

[6] Hansen K, Pancherz H. Long-term effects of Herbst treatment in relation to normal growth development: a cephalometric study. Eur J Orthod 1992;14:285-295.
[7] Lamparski DG. Skeletal age assessment utilizing cervical vertebrae. Dissertation. Pittsburgh: The University of Pittsburgh, 1972.

[8] Lavergne J, Gasson N. Operational definitions of mandibular morphogenetic and positional rotations. Scand J Dent Res 1977;85:185-192.

[9] Malmgren O, Ömblus J, Hägg U, Pancherz H. Treatment with an appliance system in relation to treatment intensity and growth periods. Am J Orthod Dentofacial Orthop 1987;91:143-151.

[10] McNamara JA Jr, Bookstein FL, Shaughnessy TG. Skeletal and dental changes following functional regulator therapy on Class II patients. Am J Orthod 1985;88:91-110.

[11] O'Reilly M, Yanniello GJ. Mandibular growth changes and maturation of cervical vertebrae-a longitudinal cephalometric study. Angle Orthod 1988;58:179-184.

[12] Pancherz H. The nature of Class II relapse after Herbst appliance treatment: a cephalometric long-term investigation. Am J Orthod Dentofacial Orthop 1991;100:220233.

[13] Pancherz H. The modern Herbst appliance. In: Graber TM, Rakosi T, Petrovic AG, eds. Dentofacial orthopedics with functional appliances. 2nd ed., St. Louis: MosbyYearbook, 1997:336-366.

[14] Pancherz H, Fackel U. The skeletofacial growth pattern pre- and post-dentofacial orthopedics. A long-term study of Class II malocclusions treated with the Herbst appliance. Eur J Orthod 1990;12:209-218.

[15] Petrovic A, Stutzmann J, Lavergne J. Mechanism of craniofacial growth and modus operandi of functional appliances: a cell-level and cybernetic approach to orthodontic decision making. In: Carlson DS, ed. Craniofacial growth theory and orthodontic treatment. Craniofacial Growth Series, Vol 23, Ann Arbor: Center for Human Growth and Development, Univ. Michigan, 1990.

[16] Sadowski PL. Craniofacial growth and the timing of treatment. Am J Orthod Dentofacial Orthop 1998;113:19-23.

[17] Toth LR, McNamara JA Jr. Skeletal and dentoalveolar adaptations produced by twin block appliance treatment. Am J Orthod Dentofacial Orthop 1999;116:597-609.

[18] White L. Early orthodontic intervention. Am J Orthod Dentofacial Orthop 1998;113:24-28. 